Wenn Kooperation endet

\title{
Lücken im Partnervertrag führen zu Streit
}

— Praxispartner sollten gleich zu Beginn ihrer Kooperation regeln, was im Fall einer Trennung geschehen soll. Andernfalls kann es zu unerquicklichem Streit und Taktiererei kommen, wie ein vom Bundesverfassungsgericht verhandelter Fall zeigt [Az.: 2 BvR 1013/11]: Die streitenden Ärzte hatten gemeinschaftlich eine Arztpraxis betrieben, an der sie jeweils zur Hälfte beteiligt waren. Als es zum Bruch kam, kündigten beide Ärzte wechselseitig, einer übernahm die Praxis samt Inventar. Geld für einen immateriellen Praxiswert (Goodwill) zahlte dieser Arzt nicht aus. Der andere verlangte daher, der in der Praxis verbliebene Arzt müsse die Abschlussbilanz, aus der der vereinbarte Goodwill hervorgeht, erstellen oder zumindest an der Abschlussbilanz mitwirken. Sein Versuch, selbst an die notwendigen Daten zu kommen, führte zum Vorwurf des Kollegen, er wolle früher gemeinsame Patienten für seine neue Praxis abwerben. Zudem behauptete der verbliebene Arzt, sein Kollege habe inzwischen über das Rechenzentrum alle für eine Abschlussbilanz notwendigen Daten bekommen.
Das Oberlandesgericht (OLG) München ignorierte dies und stützte sich einzig und allein auf die Behauptung des Klägers, er sei nicht an die notwendigen Daten gekommen. Daher verurteilte das OLG den verbliebenen Arzt zur Mitwirkung an der Abschlussbilanz. Das Bundesverfassungsgericht erklärte nun, für das OLG sei es offenkundig entscheidungserheblich gewesen, wer Zugriff auf die Daten hatte. Daher habe das Gericht den Vortrag samt Beweisangeboten nicht ignorieren dürfen, dass beiden Ärzten der Zugriff auf die Daten möglich gewesen sei. Nach dem Karlsruher Beschluss soll das OLG München das Verfahren wieder aufnehmen und neu über den Streit entscheiden. Martin Wortmann

\section{Umfrage im Gesundheitswesen}

\section{Wie gesund leben Mitarbeiter?}

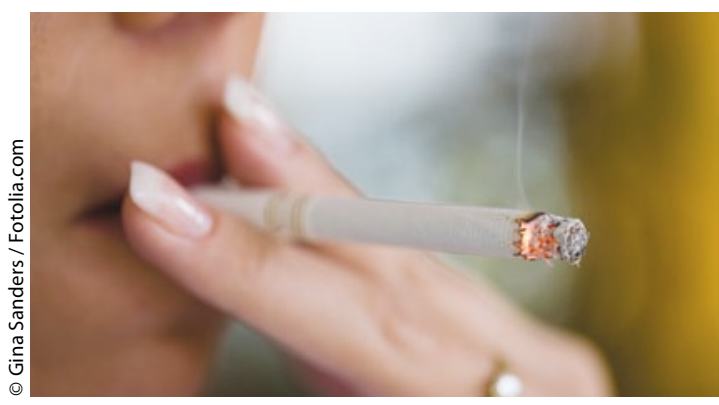

Sowohl für Patienten als auch für Ärzte gilt: „Rauchen gefährdet Ihre Gesundheit".

- Nach einer Umfrage des amerikanischen Centers for Disease Control and Prevention (CDC) verhalten sich in der Patientenversorgung tätige Berufsgruppen in vielerlei Hinsicht nicht gesundheitsbewusster als der Rest der Bevölkerung [Helfand BK, Mukamal KJ. Arch Intern Med. 2012 Dec 17:1-2. (Epub ahead of print)]. CDC-Mitarbeiter hatten 260.558 US-Amerikaner zu Präventionsverhalten und Lebensstil befragt, darunter 21.380 Personen, die direkt in der Versorgung von Patienten arbeiteten.

Bei den meisten Verhaltensweisen machte sich die Zugehörigkeit zu einem Gesundheitsberuf nicht bemerkbar. So gab es etwa bei der Teilnahme an Vorsorge-Koloskopie (64\%) und Pap-Test (82\%), beim Zahnarztbesuch (70\% im letzten Jahr), bei Übergewicht und Adipositas (64\%), beim Raucheranteil (18\%) oder bei der Häufigkeit von Sonnenbränden ( $29 \%$ im letzten Jahr) oder HIV-Risikoverhalten (5\%) keinen Unterschied zur übrigen Bevölkerung. Mehr noch: Frauen über 50, die im Gesundheitswesen tätig waren, gingen sogar seltener zur Mammografie als andere Frauen (79\%).

Beate Schumacher

\section{Schmerztherapeuten:}

\section{Geplante EBM-Reform in der Kritik}

— Kommt die EBM-Reform so wie von der Kassenärztlichen Bundesvereinigung (KBV) bisher geplant, dann würde das zu „massiven Einbrüchen in der Versorgung von Patienten mit chronischen Schmerzen " führen. Das glaubt der Berufsverband der Ärzte und Psychologischen Psychotherapeuten in der Schmerz- und Palliativmedizin in Deutschland (BVSD). „Eine weitere Honorarkürzung ist für die meisten schmerztherapeutisch tätigen Kolleginnen und Kollegen wirtschaftlich nicht mehr zu verkraften“, äußert sich BVSD-Vorsitzender Prof. Joachim Nadstawek laut Pressemitteilung. Der KBV seien die Daten zur Versorgungssituation von Patienten mit chronischen Schmerzen hinlänglich bekannt.

Die Schmerztherapeuten stören sich vor allem daran, dass sie nicht Zugang zur fachärztlichen Strukturpauschale für "grundversorgende Fachgruppen" bekommen sollen. Nicht zu dieser Gruppe zählende Fachärzte, wie die an der Qualitätssicherungsvereinbarung Schmerztherapie teilnehmenden Schmerztherapeuten, müssten mit einem rund 4\%igen Honorarabschlag rechnen, so der BVSD. Schmerztherapeuten, die Leistungen außerhalb der Schmerztherapie vorwiegend im hausärztlichen Kapitel erbringen, sollen nach den aktuellen Plänen der KBV übrigens, wie berichtet, auch nicht zu den typischen Hausärzten zählen. Bereits heute würden viele niedergelassene Schmerztherapeuten angesichts ihrer wirtschaftlichen Situation eine Aufgabe ihrer schmerztherapeutischen Tätigkeit in Betracht ziehen, heißt es weiter. „Besonders für Anästhesisten, die die Mehrzahl der Schmerztherapeuten darstellen, würde durch die EBM-Reform ein sehr starker Anreiz geschaffen, der Schmerztherapie den Rücken zu kehren. Das kann auch die KBV vor dem Hintergrund, dass in Deutschland mehr chronisch Schmerzkranke als Diabetiker leben, nicht ernsthaft wollen", so Nadstawek.

Noch 2009 habe der Erweiterte Bewertungsausschuss die Versorgung chronisch schmerzkranker Patienten als „besonders förderungswürdige Leistung “ festgestellt. Schmerztherapeutisch ausgebildete Ärzte seien festgelegten Qualitätsanforderungen unterworfen wie z.B. Fallzahlbegrenzung (max. 300 Fälle je Arzt im Quartal), organisatorischen Abläufen, Teilnahme an Schmerzkonferenzen. Nur rund 1.000 niedergelassene Schmerztherapeuten nehmen bisher an der Qualitätssicherungsvereinbarung Schmerztherapie teil. 